\title{
Bound states of the $D$-dimensional Schrödinger equation for the generalized Woods-Saxon potential
}

\author{
V. H. Badalov ${ }^{1} *$ B. Baris ${ }^{2}$, and K. Uzun ${ }^{3}$ \\ ${ }^{1}$ Institute for Physical Problems, Baku State University, \\ Z. Khalilov st. 23, AZ-1148, Baku, Azerbaijan \\ 2 Faculty of Arts and Sciences, Giresun University, 28100, Turkey \\ 3 Department of Physics, Karadeniz Technical University,61080, Trabson, Turkey \\ (Dated:)
}

\begin{abstract}
In this paper, the approximate analitical solutions of the hyper-radial Schrödinger equation are obtained for the generalized Wood-Saxon potential by implementing the Pekeris approximation to surmount the centrifugal term. The energy eigenvalues and corresponding hyper-radial wave functions are found for any angular momentum case via the Nikiforov-Uvarov (NU) and Supersymmetric quantum mechanics (SUSY QM) methods. Hence, the same expressions are obtained for the energy eigenvalues, and the expression of hyper-radial wave functions transformed each other is shown owing to these methods. Furthermore, a finite number energy spectrum depending on the depths of the potential well $V_{0}$ and $W$, the radial $n_{r}$ and $l$ orbital quantum numbers and parameters $D, a, R_{0}$ are also identified in detail. Finally, the bound state energies and the corresponding normalized hyper-radial wave functions for the neutron system of the a ${ }^{56} \mathrm{Fe}$ nucleus are calculated in $D=2$ and $D=3$, as well as the energy spectrum expressions of other highest dimensions are identified by using the energy spectrum of $D=2$ and $D=3$.

PACS numbers: 03.65.Ge, 03.65.-W, 03.65.Fd, 02.30.Gp

Keywords: Bound states, Generalized Woods-Saxon potential, Nikiforov - Uvarov method, Supersymmetric Quantum Mechanics
\end{abstract}

*Electronic address: E-mail:badalovvatan@yahoo.com 


\section{INTRODUCTION}

The Woods-Saxon potential was first proposed by R.D.Woods and D.S.Saxon in 1954 to describe the elastic scattering of $20 \mathrm{MeV}$ protons on heavy nuclei [1]. It is well known that the Woods-Saxon potential which is considered in the present study is one of the most realistic short-range potentials in physics. Since then, the Woods-Saxon potential has attracted a great deal of interest over the years and has been one of the most useful models for determining the single particle energy levels of nuclei [2 -5] and the nucleusnucleus interactions [6-10]. Furthermore, the exactly or quasi-exactly solutions of the Woods-Saxon type potential for the wave equations (Schrödinger, Dirac, Klein-Gordon) are of high scientific importance in the conceptual understanding of the interactions between the nucleon and the nucleus for both the bound and resonant states. The modified version of the Woods-Saxon potential consists of the volume (standard) Woods-Saxon and its derivative called the Woods-Saxon surface potential and is given by $[11 \llbracket 15]$ :

$$
V(r)=-\frac{V_{0}}{1+e^{\frac{r-R_{0}}{a}}}+\frac{W e^{\frac{r-R_{0}}{a}}}{\left(1+e^{\frac{r-R_{0}}{a}}\right)^{2}}, \quad\left(a<<R_{0}\right),
$$

where $V_{0}$ and $W$ represent the depths of the potential well, $R_{0}$ and $a$ are the radius of the potential and the width of the surface diffuseness, respectively. The surface term in the generalized Woods-Saxon potential induces an extra potential pocket especially at the surface region of the potential, and this pocket is so significant to explain the elastic scattering of some nuclear reactions [6-10]. Moreover, the Woods-Saxon surface potential induces a potential barrier for $W<0$ so that it may be used in the explanation of the resonant states (quasi-bound states) in nuclei. There are some special cases of the generalized Woods-Saxon potential: the GWS potential is reduced to the standard Woods-Saxon form [16] for $W=0$ and the square well potential for $W=0$ and $a \longrightarrow 0$ [16]. Furthermore the GWS potential is reduced to the Rosen-Morse potential [17] for $R_{0}=0$ [13].

The exact analytical solutions of the wave equations such as Schrödinger, Dirac and Klein-Gordon with a physical potential is of paramount importance in quantum physics since the wave function and its associated eigenvalues contain all necessary information for full description of a quantum system. It is well-known that the exact analytical solutions of the this equations are very important, since a bound states of the wave function is more con- 
venient than the wave function obtained by numerical calculation in explaining the behavior of the system under consideration. Note that, along the years, there was a huge amount of research effort to solve exactly the radial Schrödinger equation for all values of $n_{r}$ and $l$ quantum numbers. Unfortunately, it could only be possible for a very limited number of potentials such as harmonic oscillators, Coulomb, Kratzer potentials and so on [16] with a centrifugal term for $l \neq 0$. In this way, there are several established analytical methods, including Polynomial solution [16, 18, 19], Nikiforov-Uvarov method (NU) [20], Supersymmetric quantum mechanics method (SUSY QM) 21, 22], and Asymptotic iteration method (AIM) [23 29], in order to solve analytically the radial Schrödinger equation for $l \neq 0$ within various potentials. G.Levai et al. suggested a simple method for the proposed potentials for which the Schrödinger equation can be solved exactly with special functions [30] and presented relationship between the introduced formalism and supersymmetric quantum mechanics [21]. Furthermore, in order to solve the Schrödinger equation applicable to problems of non-perturbative nature, P.Amore et al. introduced a novel method [31]. Thus, this method was applied to calculate the energies and wave functions of the ground and first excited state of the quantum anharmonic potential. Although S.Flügge gave an exact expression for the wave function and suggested a graphical method for the energy eigenvalues at $l=0$, this potential could not be solved exactly without using any approximation for $l \neq 0$ yet $[16]$.

In recent years, the Nikiforov-Uvarov (NU) [20] and Supersymmetric quantum mechanics (SUSY QM) [21, 22] methods with various approximations have been proposed for solving the Schrödinger type equations analytically. Many papers show the power and simplicity of both of these methods in solving central and noncentral potentials [32-43]. Hence, NU method allows one to solve the second order linear differential equation by reducing to a generalized equation of hypergeometric type which is a second order type homogeneous differential equation with polynomials coefficients of degree not exceeding the corresponding order of differentiation, and SUSY QM method is based on determining the eigenstates of known analytically solvable potentials using algebraic operator formalism without ever having to solve the Schrödinger differential equation by standard series technique. That is why, it would be more significant to solve the non-relativistic radial Schrödinger equation with using both of these methods for the generalized Woods-Saxon potential for $l \neq 0$, since it has been extensively utilized to describe the bound and continuum states of the interacting 
systems. In this way, one can need to obtain the energy eigenvalues and corresponding eigenfunctions of the one particle problem within this potential. The NU method was used in [13] to solve the radial Schrödinger equation for the generalized Woods-Saxon potential for $l=0$. In this paper, it has been noted that there are inconsistent between the analytical and the numerical results for the GWS potential with the $l=0$ state [13]. Hence, in mentioned study, the authors made errors in application of the NU method, and these errors have led to incorrect results [44]. Later authors made also similar errors in application of the Nikiforov-Uvarov and Asymptotic iteration methods in the following works [45 48].

Note that, there are some efforts to obtain the approximate analytical solutions of the wave equations in terms of the $l \neq 0$ case; the most widely used approximation is introduced by Pekeris [49] for the exponential type potential so that this approximation is based on the expansion of the centrifugal barrier in a series of exponentials depending on the internuclear distance up to second order. Another approximation was proposed for the centrifugal term by Greene and Aldrich [50]

$$
\frac{1}{r^{2}}=\delta^{2} \frac{e^{\delta r}}{\left(1-e^{\delta r}\right)^{2}},
$$

However, this approximation is only effective for small values of the screening parameter $\delta$ [28].

In our previous works $[32-34]$, in the first time we have given the comprehensive information of how to obtain analytically the exact energy eigenvalues and the corresponding wave functions of the radial Schrödinger and the radial Klein-Gordon equations with Woods-Saxon potential via NU method. Furthermore, the hyper-radial Schrödinger equation with WoodsSaxon potential was solved within the context of the NU, AIM, and SUSY QM methods through the Pekeris approximation to the centrifugal potential, and the energy eigenvalues and corresponding radial wave functions are found for any arbitrary $l$ state as well [35]. In these works [32 35], the below approximation scheme - the Pekeris approximation [49] was proposed for $V_{l}(r)=\frac{\hbar^{2} \tilde{l}(\tilde{l}+1)}{2 \mu r^{2}}$ the centrifugal potential in any arbitrary $l$ state:

$$
\frac{1}{r^{2}}=\frac{1}{R_{0}^{2}}\left(C_{0}+\frac{C_{1}}{1+e^{\frac{r-R_{0}}{a}}}+\frac{C_{2}}{\left(1+e^{\frac{r-R_{0}}{a}}\right)^{2}}\right),
$$

where $C_{0}, C_{1}, C_{2}$ quantities dependent on $R_{0}, a$ specific potential parameters were defined 
by comparing both sides of Eq.(1.1) expression expanding in the Taylor series around the point $r=R_{0}$. But, in Ref. [51] using NU and SUSY QM methods to solve successfully the $D$-dimensional Schrödinger equation with Woods-Saxon potential by applying the Pekeris approximation to the centrifugal potential $V_{l}(r)$ based on the settings $C_{0}, C_{1}, C_{2}$ quantities which are determined around the point $r=r_{\min }$ of the effective potential $V_{\text {eff }}(r)$ in any arbitrary $l$ state. There it became clear that the system has not the bound states for $n_{r}=0$, $l=0$ when $D=3$ and $D=4$, and its reason is related with the standard Woods-Saxon potential cannot describe the system fully. As a way out of this, one need to solve this issue with utilizing the modified version of the standard Woods-Saxon potential such as the generalized Woods-Saxon potential, or the spin and pseudospin symmetries in the standard Woods-Saxon potential [52 57].

In present paper, our aim is to solve of the hyper radial Schrödinger equation for the generalized Woods-Saxon potential and to obtain the eigenvalues of energy and corresponding eigenfunctions for arbitrary $l$ angular momentum, by using the Nikiforov-Uvarov and Supersymmetric quantum mechanics methods.

The remainder of the paper is organized as follows: After this introduction, the detailed description of the $D$-dimensional SE with the generalized Woods-Saxon potential is given in Section II. Next, the implementation of Nikiforov-Uvarov (NU) and Supersymmetric quantum mechanics (SUSY QM) methods to hyper-radial SE which will be a useful guideline for investigators are presented in Section III and IV, respectively. Then in Section V, the results and discussion are presented. Finally, the paper is concluded with brief summary in Section VI.

\section{THE $D$-DIMENSIONAL SCHRÖDINGER EQUATION WITH THE WOODS- SAXON POTENTIALS}

In the $D$-dimensional $D \geq 2$ space, the Schrödinger equation with spherically symmetric potential $V(r)$ is of the form [58]

$$
\left(-\frac{\hbar^{2}}{2 \mu} \nabla_{D}^{2}+V(r)-E_{n_{r} l}\right) \psi_{n_{r} l m}\left(r, \Omega_{D}\right)=0,
$$

where $D$ is dimension of space, $\mu$ is the reduced mass, $r$ is hyperradius, $\Omega_{D}=$ $\left(\theta_{1}, \theta_{2}, \ldots, \theta_{D-2}, \phi\right)$ is hyperangular, $\hbar$ is the Planck's constant, $\triangle_{D} \equiv \nabla_{D}^{2}$ is the 
Laplasian operator and

$$
\psi_{n_{r} l m}\left(r, \Omega_{D}\right)=R_{n_{r} l}(r) Y_{l m}\left(\Omega_{D}\right)
$$

The Laplasian operator divides into a hyper-radial part $r^{1-D} \frac{\partial}{\partial r}\left(r^{D-1} \frac{\partial}{\partial r}\right)$ and an angular part $-\frac{\hat{L}_{D}^{2}}{\hbar^{2} r^{2}}$, i.e.

$$
\nabla_{D}^{2}=r^{1-D} \frac{\partial}{\partial r}\left(r^{D-1} \frac{\partial}{\partial r}\right)-\frac{\hat{L}_{D}^{2}}{\hbar^{2} r^{2}}
$$

where $\hat{L}_{D}$ is the grand orbital angular momentum operator. The eigenfunctions of $\hat{L}_{D}^{2}$ are the hyper-spherical harmonics

$$
\hat{L}_{D}^{2} Y_{l m}\left(\Omega_{D}\right)=\hbar^{2} l(l+D-2) Y_{l m}\left(\Omega_{D}\right)
$$

where $l$ is the angular momentum quantum number.

After substituting the Eqs.(2.2) - (2.4) into (2.2) and using $\psi_{n_{r} l m}\left(r, \Omega_{D}\right)$ as the eigenfunction of $\hat{L}_{D}^{2}$ with eigenvalue $\hbar^{2} l(l+D-2)$, we obtain an equation known as the hyper-radial Schrödinger equation with generalized Woods-Saxon potential

$\frac{d^{2} R_{n_{r} l}(r)}{d r^{2}}+\frac{D-1}{r} \frac{d R_{n_{r} l}(r)}{d r}+\frac{2 \mu}{\hbar^{2}}\left[E_{n l}-V(r)-\frac{\hbar^{2} l(l+D-2)}{2 \mu r^{2}}\right] R_{n_{r} l}(r)=0,(0 \leq r<\infty)$.

Introducing a new function $u_{n_{r} l}(r)=r^{\frac{D-1}{2}} R_{n_{r} l}(r)$ and a new parametr $\tilde{l}=l+\frac{D-3}{2}$, Eq.(2.5) reduces to

$$
\frac{d^{2} u_{n_{r} l}(r)}{d r^{2}}+\frac{2 \mu}{\hbar^{2}}\left[E_{n_{r} l}-V_{e f f}(r)\right] u_{n_{r} l}(r)=0,
$$

where $V_{\text {eff }}(r)$ is effective potential, i.e.

$$
V_{e f f}(r)=V(r)+\frac{\hbar^{2} \tilde{l}(\tilde{l}+1)}{2 \mu r^{2}} .
$$

Equation (2.7) has the same form as the equation for a particle in one dimension, except for two important differences. First, there is a repulsive effective potential proportional to the eigenvalue of $\hbar^{2} \tilde{l}(\tilde{l}+1)$. Second, the radial function must satisfy the boundary conditions $u(0)=0$ and $u(\infty)=0$. 
It is well-known that the hyper-radial Schrödinger equation cannot be solved exactly for this potential at the value $l \neq 0$ by using the standard methods as SUSY QM, AIM, NU and so on. From Eq.(2.7), it is seen that the effective potential is combination of the exponential and inverse square potentials which cannot be solved analytically. That is why, the Pekeris approximation which is the most widely used and convenient for our purposes can be taken in order to solve this issue. This approximation is based on the expansion the series for exponential cases depending on the internuclear distance of the centrifugal barrier, and there the terms up to second order are considering.

After introducing new variable $x=\frac{r-R_{0}}{R_{0}}$ or $r=R_{0}(1+x)$, the generalized Woods-Saxon potential in that case has the following form:

$$
V(r)=-\frac{V_{0}}{1+e^{\alpha x}}+\frac{W e^{\alpha x}}{\left(1+e^{\alpha x}\right)^{2}}
$$

where $\alpha=\frac{R_{0}}{a}$. The extreme point of the generalized Woods-Saxon potential $V(r)$ is defined as

$$
\exp (\alpha x)=\frac{W-V_{0}}{W+V_{0}} \quad \text { or } \quad \exp \left(\frac{r-R_{0}}{a}\right)=\frac{W-V_{0}}{W+V_{0}} .
$$

and their solution of Eq.(2.9) is the following form:

$$
x=x_{\text {min }}=\frac{1}{\alpha} \ln \left(\frac{W-V_{0}}{W+V_{0}}\right) \quad \text { or } \quad r=r_{\text {min }}=R_{0}+a \ln \left(\frac{W-V_{0}}{W+V_{0}}\right) .
$$

It must be noted, there the conditions $W>V_{0}$ and $W<-V_{0}$ is obtained based on the relation $\exp (\alpha x)=\frac{W-V_{0}}{W+V_{0}}$ because $\exp (\alpha x)>0$ in an arbitrary value of $x$. If this condition is not satisfied, there are not minimum point in the generalized Woods-Saxon potential $V(r)$.

Let us expand centrifugal potential $V_{l}(r)$ in Taylor series around the point of $x=x_{\text {min }}=$ $x_{e}\left(r=r_{\min }=r_{e}\right)$ :

$$
\begin{gathered}
V_{l}(r)=\frac{\hbar^{2} \tilde{l}(\tilde{l}+1)}{2 \mu r^{2}}=\frac{\hbar^{2} \tilde{l}(\tilde{l}+1)}{2 \mu R_{0}^{2}} \cdot \frac{\tilde{\delta}}{(1+x)^{2}}=\tilde{\delta}\left[\frac{1}{\left(1+x_{l}\right)^{2}}-\frac{2}{\left(1+x_{l}\right)^{3}} \cdot\left(x-x_{l}\right)+\right. \\
\left.+\frac{3}{\left(1+x_{l}\right)^{3}} \cdot\left(x-x_{l}\right)^{2}+o\left(\left(x-x_{l}\right)^{3}\right)\right],
\end{gathered}
$$

where $\tilde{\delta}=\frac{\hbar^{2} \tilde{l}(\tilde{l}+1)}{2 \mu R_{0}^{2}}$. By using the Pekeris approximation, $V_{l}(r)$ takes the form $[32,35$, , 51]

$$
\tilde{V}_{l}(r)=\tilde{\delta}\left(C_{0}+\frac{C_{1}}{1+e^{\alpha x}}+\frac{C_{2}}{\left(1+e^{\alpha x}\right)^{2}}\right)
$$


Let us expand the potential $\tilde{V}_{l}(r)$ in the Taylor series around the point of $x=x_{\min }=x_{e}$ $\left(r=r_{\min }=r_{e}\right)$ :

$$
\begin{aligned}
\tilde{V}_{l}(x) & =\tilde{\delta}\left[C_{0}+\frac{C_{1}}{1+e^{\alpha x_{e}}}+\frac{C_{2}}{\left(1+e^{\alpha x_{e}}\right)^{2}}-\left(\frac{\alpha C_{1} e^{\alpha x_{e}}}{\left(1+e^{\alpha x_{e}}\right)^{2}}+\frac{2 \alpha C_{2} e^{\alpha x_{e}}}{\left(1+e^{\alpha x_{e}}\right)^{3}}\right)\left(x-x_{e}\right)+\right. \\
& \left.-\left(\frac{\alpha^{2} C_{1} e^{\alpha x_{e}}\left(1-e^{\alpha x_{e}}\right)}{2\left(1+e^{\alpha x_{e}}\right)^{3}}+\frac{\alpha^{2} C_{2} e^{\alpha x_{e}}\left(1-2 e^{\alpha x_{e}}\right)}{\left(1+e^{\alpha x_{e}}\right)^{4}}\right)\left(x-x_{e}\right)^{2}+o\left(\left(x-x_{e}\right)^{3}\right)\right]
\end{aligned} .
$$

In order to define the constants $C_{0}, C_{1}$ and $C_{2}$, we should compare the compatible degrees of same order of $x$ in Eqs.(2.11) and (2.13), and we get the following algebraic set of equations:

$$
\left\{\begin{array}{l}
C_{0}+\frac{C_{1}}{1+e^{\alpha x_{e}}}+\frac{C_{2}}{\left(1+e^{\alpha x_{e}}\right)^{2}}=\frac{1}{\left(1+x_{e}\right)^{2}} \\
\frac{\alpha C_{1} e^{\alpha x_{e}}}{\left(1+e^{\alpha x_{e}}\right)^{2}}+\frac{2 \alpha C_{2} e^{\alpha x_{e}}}{\left(1+e^{\alpha x_{e}}\right)^{3}}=\frac{2}{\left(1+x_{e}\right)^{3}} \\
\frac{\alpha^{2} C_{1} e^{\alpha x_{e}}\left(1-e^{\alpha x_{e}}\right)}{2\left(1+e^{\alpha x_{e}}\right)^{3}}+\frac{\alpha^{2} C_{2} e^{\alpha x_{e}}\left(1-2 e^{\alpha x_{e}}\right)}{\left(1+e^{\alpha x_{e}}\right)^{4}}=-\frac{3}{\left(1+x_{e}\right)^{4}}
\end{array} .\right.
$$

From the Eq.(2.14), for $C_{0}, C_{1}$ and $C_{2}$ constants is obtained as:

$$
\left\{\begin{array}{l}
C_{0}=\frac{1}{\left(1+x_{e}\right)^{2}}+\frac{\left(1+e^{\alpha x_{e}}\right)^{2}}{\alpha e^{\alpha x}\left(1+x_{e}\right)^{3}}\left[\frac{e^{-\alpha x_{e}}-3}{1+e^{\alpha x_{e}}}+\frac{3 e^{-\alpha x_{e}}}{\alpha\left(1+x_{e}\right)}\right] \\
C_{1}=\frac{2\left(1+e^{\alpha x_{e}}\right)^{2}}{\alpha e^{\alpha x_{e}\left(1+x_{l}\right)^{3}}}\left[2-e^{-\alpha x_{e}}-\frac{3\left(1+e^{-\alpha x_{e}}\right)}{\alpha\left(1+x_{e}\right)}\right] \\
C_{2}=\frac{\left(1+e^{\alpha x_{e}}\right)^{3}}{\alpha e^{\alpha x_{e}}\left(1+x_{e}\right)^{3}}\left[e^{-\alpha x_{e}}-1+\frac{3\left(1+e^{-\alpha x_{e}}\right)}{\alpha\left(1+x_{e}\right)}\right]
\end{array}\right.
$$

After Pekeris approximation, the effective potential takes as following form:

$$
\tilde{V}_{e f f}(r)=V_{W S}(r)+\tilde{V}_{l}(r)=\tilde{\delta} C_{0}-\frac{V_{0}+W-\tilde{\delta} C_{1}}{1+e^{\frac{r-R_{0}}{a}}}+\frac{W+\tilde{\delta} C_{2}}{\left(1+e^{\frac{r-R_{0}}{a}}\right)^{2}} .
$$

If we consider $x_{e}=0$ in Eq.(2.15) relations, the constants $C_{0}, C_{1}$ and $C_{2}$ becomes in the following form $32-35]$ :

$$
C_{0}=1-\frac{4}{\alpha}+\frac{12}{\alpha^{2}}, C_{1}=\frac{8}{\alpha}-\frac{48}{\alpha^{2}}, C_{2}=\frac{48}{\alpha^{2}} .
$$

Instead of solving the hyper-radial Schrödinger equation for the effective generalized Woods-Saxon potential $V_{\text {eff }}(r)$ given by Eq.(2.7), we now solve the radial Schrödinger equation for the new effective potential $\tilde{V}_{e f f}(r)$ given by Eq.(2.16) obtained using the Pekeris approximation. Having inserted this new effective potential into Eq.(2.6), we obtain

$$
\frac{d^{2} u_{n_{r} l}(r)}{d r^{2}}+\frac{2 \mu}{\hbar^{2}}\left[E_{n_{r} l}-\tilde{\delta} C_{0}+\frac{V_{0}+W-\tilde{\delta} C_{1}}{1+e^{\frac{r-R_{0}}{a}}}-\frac{W+\tilde{\delta} C_{2}}{\left(1+e^{\frac{r-R_{0}}{a}}\right)^{2}}\right] u_{n_{r} l}(r)=0
$$


If the Eq.(2.18) is rewrite by using a new variable of the form $z=\left(1+e^{\frac{r-R_{0}}{a}}\right)^{-1}$, it will be obtained as

$$
z^{2}(1-z)^{2} u^{\prime \prime}(z)+z(1-z)(1-2 z) u^{\prime}(z)+\frac{2 \mu a^{2}}{\hbar^{2}}\left[E-\tilde{\delta} C_{0}+\left(V_{0}+W-\tilde{\delta} C_{1}\right) z-\left(W+\tilde{\delta} C_{2}\right) z^{2}\right] u(z)=0
$$

If we use the following dimensionless notations

$$
\varepsilon^{2}=-\frac{2 \mu a^{2}\left(E-\tilde{\delta} C_{0}\right)}{\hbar^{2}}>0, \beta^{2}=\frac{2 \mu a^{2}\left(V_{0}+W-\tilde{\delta} C_{1}\right)}{\hbar^{2}}>0, \gamma^{2}=\frac{2 \mu a^{2}\left(W+\tilde{\delta} C_{2}\right)}{\hbar^{2}}>0
$$

we will get

$$
u^{\prime \prime}(z)+\frac{1-2 z}{z(1-z)} u^{\prime}(z)+\frac{-\varepsilon^{2}+\beta^{2} z-\gamma^{2} z^{2}}{(z(1-z))^{2}} u(z)=0, \quad(0 \leq z \leq 1)
$$

with real $\varepsilon>0(E<0)$ for bound states; $\beta$ and $\gamma$ are real and positive.

\section{SOLUTION OF THE HYPER-RADIAL SCHRÖDINGER EQUATION BY NIKIFOROV-UVAROV METHOD}

According to the NU-method [20], the Eq.(2.21) is compared with the equation of $\psi^{\prime \prime}(z)+$ $\frac{\tilde{\tau}(z)}{\sigma(z)} \psi^{\prime}(z)+\frac{\tilde{\sigma}(z)}{\sigma^{2}(z)} \psi(z)=0$, and the corresponding polinomials are obtained

$$
\tilde{\tau}(z)=1-2 z ; \sigma(z)=z(1-z) ; \tilde{\sigma}(z)=-\varepsilon^{2}+\beta^{2} z-\gamma^{2} z^{2}
$$

The new function $\pi(z)=\frac{\sigma^{\prime}(z)-\tau \tilde{(z)}}{2} \pm \sqrt{\left(\frac{\sigma^{\prime}(z)-\tau \tilde{(z)}}{2}\right)^{2}-\sigma \tilde{(z)}+k \sigma(z)}$ in Ref. [20] can be found by substituting Eq.(3.1) and taking $\sigma^{\prime}(z)=1-2 z$. Hence, the function $\pi(z)$ is

$$
\pi(z)= \pm \sqrt{\varepsilon^{2}+\left(k-\beta^{2}\right) z-\left(k-\gamma^{2}\right) z^{2}} .
$$

Further, the constant parameter $k$ can be found by utilizing the condition that the expression under the square root has a double zero, i.e., its discriminant is equal to zero. Therefore, the parameter $k$ can be obtained as

$$
k=\beta^{2}-2 \varepsilon^{2} \pm 2 \varepsilon \sqrt{\varepsilon^{2}-\beta^{2}+\gamma^{2}} .
$$


So, we arrive at the following four possible functions of $\pi(z)$

$$
\pi(z)= \begin{cases}\left(\varepsilon-\sqrt{\varepsilon^{2}-\beta^{2}+\gamma^{2}}\right) z-\varepsilon, & \text { for } k=\beta^{2}-2 \varepsilon^{2}+2 \varepsilon \sqrt{\varepsilon^{2}-\beta^{2}+\gamma^{2}} \\ -\left(\varepsilon+\sqrt{\varepsilon^{2}-\beta^{2}+\gamma^{2}}\right) z+\varepsilon, & \text { for } k=\beta^{2}-2 \varepsilon^{2}+2 \varepsilon \sqrt{\varepsilon^{2}-\beta^{2}+\gamma^{2}} \\ \left(\varepsilon+\sqrt{\varepsilon^{2}-\beta^{2}+\gamma^{2}}\right) z-\varepsilon, & \text { for } k=\beta^{2}-2 \varepsilon^{2}-2 \varepsilon \sqrt{\varepsilon^{2}-\beta^{2}+\gamma^{2}} \\ -\left(\varepsilon+\sqrt{\varepsilon^{2}-\beta^{2}+\gamma^{2}}\right) z+\varepsilon, & \text { for } k=\beta^{2}-2 \varepsilon^{2}-2 \varepsilon \sqrt{\varepsilon^{2}-\beta^{2}+\gamma^{2}}\end{cases}
$$

Further, from the four possible forms of the function $\pi(z)$ we select the one for which the function $\tau(z)=\tilde{\tau}(z)+2 \pi(z)$ has the negative derivative $\tau^{\prime}(z)<0$ [20] and root lies in the interval $(0,1)$. Hence, we can select the parameter $k$ and the function $\pi(z)$ to be

$$
k=\beta^{2}-2 \varepsilon^{2}-2 \varepsilon \sqrt{\varepsilon^{2}-\beta^{2}+\gamma^{2}}
$$

and

$$
\pi(z)=\varepsilon-\left(\varepsilon+\sqrt{\varepsilon^{2}-\beta^{2}+\gamma^{2}}\right) z,
$$

which give the physicaly valid expression for the function $\tau(z)$ :

$$
\tau(z)=1+2 \varepsilon-2\left(1+\varepsilon+\sqrt{\varepsilon^{2}-\beta^{2}+\gamma^{2}}\right) z, \tau^{\prime}(z)=-2\left(1+\varepsilon+\sqrt{\varepsilon^{2}-\beta^{2}+\gamma^{2}}\right)<0 .
$$

Then, by using the relations [20] $\lambda=k+\pi^{\prime}(z)$ and $\lambda=\lambda_{n}=-n \tau^{\prime}-\frac{n(n-1)}{2} \sigma^{\prime \prime}, \quad(n=$ $0,1,2, \ldots)$ the following expressions for the constant $\lambda$ are obtained, respectively:

$$
\begin{gathered}
\lambda=\beta^{2}-2 \varepsilon^{2}-2 \varepsilon \sqrt{\varepsilon^{2}-\beta^{2}+\gamma^{2}}-\varepsilon-\sqrt{\varepsilon^{2}-\beta^{2}+\gamma^{2}}, \\
\lambda=\lambda_{n_{r}}=2\left(\varepsilon+\sqrt{\varepsilon^{2}-\beta^{2}+\gamma^{2}}\right) n_{r}+n_{r}\left(n_{r}+1\right) .
\end{gathered}
$$

After comparing the Eq.(3.8) with Eq.(3.9)

$$
\beta^{2}-2 \varepsilon^{2}-2 \varepsilon \sqrt{\varepsilon^{2}-\beta^{2}+\gamma^{2}}-\varepsilon-\sqrt{\varepsilon^{2}-\beta^{2}+\gamma^{2}}=2\left(\varepsilon+\sqrt{\varepsilon^{2}-\beta^{2}+\gamma^{2}}\right) n_{r}+n_{r}\left(n_{r}+1\right)
$$

we get

$$
\varepsilon+\sqrt{\varepsilon^{2}-\beta^{2}+\gamma^{2}}+n_{r}+\frac{1}{2}-\frac{\sqrt{1+4 \gamma^{2}}}{2}=0
$$

or

$$
\varepsilon+\sqrt{\varepsilon^{2}-\beta^{2}+\gamma^{2}}=n^{\prime} .
$$


Here

$$
n^{\prime}=-n_{r}+\frac{\sqrt{1+4 \gamma^{2}}-1}{2}
$$

$n_{r}$ is the radial quantum number $\left(n_{r}=0,1,2, \ldots\right)$. From Eq.(3.11), one can find

$$
\varepsilon=\frac{1}{2}\left(n^{\prime}+\frac{\beta^{2}-\gamma^{2}}{n^{\prime}}\right) .
$$

From the bound states $E<0$ and finite wave function, we obtain $\varepsilon>0$ and $\varepsilon^{2}-\beta^{2}+\gamma^{2}>$ 0, i.e. $n^{\prime}>0, n^{\prime}-\varepsilon>0$ and $\left|\beta^{2}-\gamma^{2}\right|<n^{\prime 2}$. Hence, using Eqs.(3.12) and (2.20) there relations can be replaced into the form:

$$
\begin{gathered}
0 \leq n_{r}<\frac{1}{2}\left(\sqrt{1+\frac{8 \mu a^{2} W}{\hbar^{2}}+\frac{4 \tilde{l}(\tilde{l}+1) a^{2} C_{2}}{R_{0}^{2}}}-1\right), \\
\left|V_{0}-\frac{\hbar^{2} \tilde{l}(\tilde{l}+1)}{2 \mu R_{0}^{2}}\left(C_{1}+C_{2}\right)\right|<\frac{\hbar^{2}}{2 \mu a^{2}}\left[\frac{1}{2}\left(\sqrt{1+\frac{8 \mu a^{2} W}{\hbar^{2}}+\frac{4 \tilde{l}(\tilde{l}+1) a^{2} C_{2}}{R_{0}^{2}}-1}\right)-n_{r}\right]_{(3.1}^{2}, \\
V_{0} R_{0}^{2} \geq \frac{\hbar^{2} \tilde{l}(\tilde{l}+1)}{2 \mu}\left(C_{0}+C_{1}+C_{2}\right) .
\end{gathered}
$$

Upon substituting with the values of $\varepsilon, \beta, \gamma, n^{\prime}$ and $\tilde{\delta}$ into Eq.(3.13) for energy eigenvalues $E_{n_{r} l}^{(D)}$, we obtain

$$
\begin{aligned}
E_{n_{r} l}^{(D)} & =\frac{V_{0}}{2}-\frac{\hbar^{2} \tilde{l}(\tilde{l}+1)}{2 \mu R_{0}^{2}}\left(C_{0}+\frac{C_{1}+C_{2}}{2}\right)-\frac{\hbar^{2}}{2 \mu a^{2}}\left[\frac{1}{16}\left(\sqrt{1+\frac{8 \mu a^{2} W}{\hbar^{2}}+\frac{4 \tilde{l}(\tilde{l}+1) a^{2} C_{2}}{R_{0}^{2}}}-2 n_{r}-1\right)^{2}+\right. \\
& \left.\frac{\left(\frac{2 \mu a^{2}}{\hbar^{2}}\right)^{2}\left[V_{0}-\frac{\hbar^{2} \tilde{l}(\tilde{l}+1)}{2 \mu R_{0}^{2}}\left(C_{1}+C_{2}\right)\right]^{2}}{\left(\sqrt{1+\frac{8 \mu a^{2} W}{\hbar^{2}}+\frac{4 \tilde{l}(\tilde{l}+1) a^{2} C_{2}}{R_{0}^{2}}}-2 n_{r}-1\right)^{2}}\right]
\end{aligned}
$$

If the conditions Eqs. (3.14) - (3.16) are satisfied simultaneously, the bound states will exist. Thus, the energy spectrum Eq. (3.17) is limited, i.e. we have only the finite number of energy eigenvalues.

For very large $V_{0}$, the $l$-dependent effective potential has the same form as the potential with $l=0$. According to Eq. (3.17) the energy eigenvalues depend on the depths of the potential well $V_{0}$ and $W$, the width $R_{0}$ potential, the thickness $a$ surface, and $D$ parameter. 
If constraints imposed on $n_{r}, V_{0}$ and $E_{n_{r} l}^{(D)}$ satisfied, the bound states appear. Any energy eigenvalue has to satisfy the following inequality: $V_{e f f, \min }<E_{n_{r} l}^{(D)}<V_{l}$, where $V_{l}=-V_{0}+$ $\frac{\hbar^{2} \tilde{l}(\tilde{l}+1)}{2 \mu R_{0}^{2}}\left(C_{0}+C_{1}+C_{2}\right)$. From Eq.(3.16) is seen that the potential depth $V_{0}$ increases when the parameter $R_{0}$ is decreasing for given $l$ quantum number and vice versa. Therefore, one can say that the bound states exist within this potential.

In addition, we have seen that there are some restrictions on the potential parameters for the bound state solutions within the framework of quantum mechanics. Hence, when the values of the parameters $n_{r}, V_{0}$ and energy eigenvalues $E_{n_{r} l}^{(D)}$ satisfy the conditions in Eqs.(3.14) - (3.16) and $V_{e f f, \text { min }}<E_{n_{r} l}^{(D)}<V_{l}$ respectively, we obtain the bound states. We also point out that the exact results obtained for the generalized Woods-Saxon potential may have some interesting applications for studying different quantum mechanical and nuclear scattering problems. Consequently, the found wave functions are physical ones.

According to the Eq. (3.17), when $l=0$ and $D=3$, the energy eigenvalues are obtained the following expression:

$$
E_{n_{r}}=\frac{V_{0}}{2}-\frac{\hbar^{2}}{2 \mu a^{2}}\left[\frac{1}{16}\left(\sqrt{1+\frac{8 \mu a^{2} W}{\hbar^{2}}}-2 n_{r}-1\right)^{2}+\frac{\left(\frac{2 \mu a^{2} V_{0}}{\hbar^{2}}\right)^{2}}{\left(\sqrt{1+\frac{8 \mu a^{2} W}{\hbar^{2}}}-2 n_{r}-1\right)^{2}}\right]
$$

It should be noted that the Eq. (3.18) is same with the energy eigenvalues expression in Ref. [44]. There the any energy eigenvalues must be less than $-V_{0}$ and bigger than the minimum value of generalized Woods-Saxon potential $V_{\min }$, i.e. $V_{\min }<E_{n_{r}}<-V_{0}$, where $V_{\text {min }}=-\frac{\left(W+V_{0}\right)^{2}}{4 W}$.

Now, we are going to define the radial eigenfunctions of this potential. Having substituted $\pi(z)$ and $\sigma(z)$ into the equations [20]

$$
\frac{\Phi^{\prime}(z)}{\Phi(z)}=\frac{\pi(z)}{\sigma(z)} \quad \text { and } \quad \frac{d}{d z}(\sigma(z) \rho(z))=\tau(z) \rho(z)
$$

one can find the finite function $\Phi(z)$ and weight function $\rho(z)$ in the interval $(0,1)$ :

$$
\begin{gathered}
\Phi(z)=z^{\varepsilon}(1-z)^{\sqrt{\varepsilon^{2}-\beta^{2}+\gamma^{2}}}, \\
\rho(z)=z^{2 \varepsilon}(1-z)^{2 \sqrt{\varepsilon^{2}-\beta^{2}+\gamma^{2}}} .
\end{gathered}
$$


By substituting the functions $\sigma(z)$ and $\rho(z)$ into Rodrigues relation [20] $y_{n}(z)=$ $\frac{B_{n}}{\rho(z)} \frac{d^{n}}{d z^{n}}\left[\sigma^{n}(z) \rho(z)\right]$, we get

$$
y_{n_{r}}(z)=B_{n_{r}} z^{-2 \varepsilon}(1-z)^{-2 \sqrt{\varepsilon^{2}-\beta^{2}+\gamma^{2}}} \frac{d^{n_{r}}}{d z^{n_{r}}}\left[z^{n_{r}+2 \varepsilon}(1-z)^{n_{r}+2 \sqrt{\varepsilon^{2}-\beta^{2}+\gamma^{2}}}\right]
$$

where $B_{n_{r}}$ is the normalization constant and its value is $\frac{1}{n_{r} !}$ [59]. After using the following definition of the Jacobi polynomial

$$
P_{n}^{(\alpha, \beta)}(1-2 z)=\frac{1}{n !} z^{-\alpha}(1-z)^{-\beta} \frac{d^{n}}{d z^{n}}\left[z^{n+\alpha}(1-z)^{n+\beta}\right]
$$

we are able to write Eq.(3.21) as

$$
y_{n_{r}}(z)=P_{n_{r}}^{\left(2 \varepsilon, 2 \sqrt{\varepsilon^{2}-\beta^{2}+\gamma^{2}}\right)}(1-2 z)
$$

According to the relation $u(z)=\Phi(z) y(z)$ [20], we obtain the hyper-radial wave functions as:

$$
u_{n_{r} l}(z)=C_{n_{r} l} z^{\varepsilon}(1-z)^{\sqrt{\varepsilon^{2}-\beta^{2}+\gamma^{2}}} P_{n_{r}}^{\left(2 \varepsilon, 2 \sqrt{\varepsilon^{2}-\beta^{2}+\gamma^{2}}\right)}(1-2 z) .
$$

Finally, the normalization constant $C_{n_{r} l}$ is determined by using the following orthogonality condition:

$$
\int_{0}^{\infty}\left|R_{n_{r} l}(r)\right|^{2} r^{D-1} d r=\int_{0}^{\infty}\left|u_{n_{r} l}(r)\right|^{2} d r=a \int_{0}^{1} \frac{\left|u_{n_{r} l}(z)\right|^{2}}{z(1-z)} d z=1
$$

\section{SOLUTION OF THE HYPER-RADIAL SCHRÖDINGER EQUATION BY SU- PERSYMMETRIC QUANTUM MECHANICS METHOD}

According to Supersymmetric quantum mechanics (SUSY QM), the eigenfunction of ground state $u_{0}(r)$ in Eq.(2.18) is a form as below [21]

$$
u_{0}(r)=N \exp \left(-\frac{\sqrt{2 \mu}}{\hbar} \int W(r) d r\right)
$$

where $N$ and $W(r)$ are normalized constant and superpotential, respectively. The connection between the supersymmetric partner potentials $V_{1}(r)$ and $V_{2}(r)$ of the superpotential $W(r)$ is as follows [21]: 


$$
V_{1}(r)=W^{2}(r)-\frac{\hbar}{\sqrt{2 \mu}} W^{\prime}(r)+E, \quad V_{2}(r)=W^{2}(r)+\frac{\hbar}{\sqrt{2 \mu}} W^{\prime}(r)+E .
$$

The particular solution of the Riccati equation Eq.(4.2) searches the following form:

$$
W(r)=-\frac{\hbar}{\sqrt{2 \mu}}\left(A+\frac{B}{1+e^{\frac{r-R_{0}}{a}}}\right),
$$

where $A$ and $B$ are unknown constants. Since $V_{1}(r)=\tilde{V}_{\text {eff }}(r)$, inserting the relations Eq.(2.16) and Eq.(4.3) into the expression Eq.(4.2), and from comparison of compatible quantities in the left and right sides of the equation, we obtain the following relations for determining $A$ and $B$ constants:

$$
A^{2}=-\frac{2 \mu}{\hbar^{2}}\left(E_{0}-\tilde{\delta} C_{0}\right), \quad 2 A B-\frac{B}{a}=-\frac{2 \mu\left(V_{0}+W-\tilde{\delta} C_{1}\right)}{\hbar^{2}}, \quad B^{2}+\frac{B}{a}=\frac{2 \mu\left(W+\tilde{\delta} C_{2}\right)}{\hbar^{2}}
$$

So, substituting Eqs.(2.20) into Eqs.(4.4), yields:

$$
A^{2}=\frac{\varepsilon^{2}}{a^{2}}, \quad 2 A B-\frac{B}{a}=-\frac{\beta^{2}}{a^{2}}, \quad B^{2}+\frac{B}{a}=\frac{\gamma^{2}}{a^{2}} .
$$

Having inserted Eq.(4.3) into Eq.(4.1), we find the eigenfunction for ground state as

$$
u_{0}(r)=N e^{A r}\left(1+e^{-\frac{r-R_{0}}{a}}\right)^{-a B} .
$$

$A$ must be less than zero $(A<0)$, and $B$ must be greater than zero $(B>0)$ for the hyperradial $u_{0}(r)$ wave function satisfy the boundary conditions $u_{0}(0)=0$ and $u_{0}(\infty)=0$. Under this circumstance, from the second and third equations Eqs.(4.5), we obtain:

$$
\begin{gathered}
A=\frac{1}{2 a}-\frac{\beta^{2}}{a\left(\sqrt{1+4 \gamma^{2}}-1\right)}, \\
B=\frac{\sqrt{1+4 \gamma^{2}}-1}{2 a},
\end{gathered}
$$

By using Eq.(4.7) and the first equation of Eq.(4.5), we will obtain the ground state energy $E_{0}^{(D)}$ as

$$
E_{0}^{(D)}=\tilde{\delta} C_{0}-\frac{\hbar^{2}}{2 \mu}\left[\frac{1}{2 a}-\frac{\beta^{2}}{a\left(\sqrt{1+4 \gamma^{2}}-1\right)}\right]^{2}
$$


When $r \rightarrow \infty$, the chosen superpotential $W(r)$ is $W(r) \rightarrow-\frac{\hbar A}{\sqrt{2 \mu}}$. Having inserted the Eq.(4.3) into Eq.(4.2), for supersymmetric partner potentials, gives:

$$
V_{1}(r)=\frac{\hbar^{2}}{2 \mu}\left[A^{2}+\frac{B^{2}+\frac{B}{a}}{\left(1+e^{\frac{r-R_{0}}{a}}\right)^{2}}+\frac{2 A B-\frac{B}{a}}{1+e^{\frac{r-R_{0}}{a}}}\right]
$$

and

$$
V_{2}(r)=\frac{\hbar^{2}}{2 \mu}\left[A^{2}+\frac{B^{2}-\frac{B}{a}}{\left(1+e^{\frac{r-R_{0}}{a}}\right)^{2}}+\frac{2 A B+\frac{B}{a}}{1+e^{\frac{r-R_{0}}{a}}}\right] .
$$

If we add side-by-side the second equation of Eqs.(4.5) to third equation of Eqs.(4.5), we will obtain:

$$
2 A B+B^{2}=\frac{\gamma^{2}-\beta^{2}}{a^{2}}
$$

from here

$$
A=\frac{\gamma^{2}-\beta^{2}}{2 a^{2} B}-\frac{B}{2}
$$

Two partner potentials $V_{1}(r)$ and $V_{2}(r)$ which differ from each other with additive constants and have the same functional form are called invariant potentials [60, 61]. Thus, for the partner potentials $V_{1}(r)$ and $V_{2}(r)$ given with Eqs.(4.10) and (4.11), the invariant forms $R\left(B_{i},\left(i=1,2, \ldots, n_{r}\right)\right.$, which are independent of $r$, has a form as below:

$$
\begin{gathered}
R\left(B_{1}\right)=V_{2}(B, r)-V_{1}\left(B_{1}, r\right)=-\frac{\hbar^{2}}{2 \mu}\left[\left(\frac{\gamma^{2}-\beta^{2}}{2 a^{2}\left(B-\frac{1}{a}\right)}-\frac{B-\frac{1}{a}}{2}\right)^{2}-\left(\frac{\gamma^{2}-\beta^{2}}{2 a^{2} B}-\frac{B}{2}\right)^{2}\right], \\
R\left(B_{i}\right)=V_{2}\left[B-\frac{i-1}{a}, r\right]-V_{1}\left[B-\frac{i}{a}, r\right]= \\
-\frac{\hbar^{2}}{2 \mu}\left[\left(\frac{\gamma^{2}-\beta^{2}}{2 a^{2}\left(B-\frac{i}{a}\right)}-\frac{B-\frac{i}{a}}{2}\right)^{2}-\left(\frac{\gamma^{2}-\beta^{2}}{2 a^{2}\left(B-\frac{i-1}{a}\right)}-\frac{B-\frac{i-1}{a}}{2}\right)^{2}\right] .
\end{gathered}
$$

If we continue this procedure and make the substitution $B_{n_{r}}=B_{n_{r}-1}-\frac{1}{a}=B-\frac{n_{r}}{a}$ at every step until $B_{n_{r}} \geq 0$, the whole discrete spectrum of Hamiltonian $H_{-}(B)$ :

$$
\begin{aligned}
& E_{n_{r} l}^{(D)}=E_{0}^{(D)}+\sum_{i=1}^{n_{r}} R\left(B_{i}\right)=\tilde{\delta} C_{0}-\frac{\hbar^{2}}{2 \mu}\left[\left(\frac{\gamma^{2}-\beta^{2}}{2 a^{2}\left(B-\frac{n_{r}}{a}\right)}-\frac{B-\frac{n_{r}}{a}}{2}\right)^{2}-\left(\frac{\gamma^{2}-\beta^{2}}{2 a^{2}\left(B-\frac{n_{r}-1}{a}\right)}-\frac{B-\frac{n_{r}-1}{a}}{2}\right)^{2}+\right. \\
& \left(\frac{\gamma^{2}-\beta^{2}}{2 a^{2}\left(B-\frac{n_{r}-1}{a}\right)}-\frac{B-\frac{n_{r}-1}{a}}{2}\right)^{2}-\left(\frac{\gamma^{2}-\beta^{2}}{2 a^{2}\left(B-\frac{n_{r}-2}{a}\right)}-\frac{B-\frac{n_{r}-2}{a}}{2}\right)^{2}+\ldots+\left(\frac{\gamma^{2}-\beta^{2}}{2 a^{2}\left(B-\frac{2}{a}\right)}-\frac{B-\frac{2}{a}}{2}\right)^{2}- \\
& \left.\left(\frac{\gamma^{2}-\beta^{2}}{2 a^{2}\left(B-\frac{1}{a}\right)}-\frac{B-\frac{1}{a}}{2}\right)^{2}+\left(\frac{\gamma^{2}-\beta^{2}}{2 a^{2}\left(B-\frac{1}{a}\right)}-\frac{B-\frac{1}{a}}{2}\right)^{2}-\left(\frac{\gamma^{2}-\beta^{2}}{2 a^{2} B}-\frac{B}{2}\right)^{2}+\left(\frac{1}{2 a}-\frac{\beta^{2}}{a\left(\sqrt{1+4 \gamma^{2}}-1\right)}\right)^{2}\right] \\
& =\tilde{\delta} C_{0}-\frac{\hbar^{2}}{2 \mu}\left[\frac{\gamma^{2}-\beta^{2}}{2 a^{2}\left(B-\frac{n_{r}}{a}\right)}-\frac{B-\frac{n_{r}}{a}}{2}\right]^{2}=\tilde{\delta} C_{0}-\frac{\hbar^{2}}{2 \mu a^{2}}\left[\frac{\beta^{2}-\gamma^{2}}{\sqrt{1+4 \gamma^{2}}-2 n_{r}-1}+\frac{\sqrt{1+4 \gamma^{2}}-2 n_{r}-1}{4}\right]^{2},
\end{aligned}
$$


and we obtain:

$$
E_{n_{r} l}^{(D)}=\tilde{\delta} C_{0}-\frac{\hbar^{2}}{2 \mu a^{2}}\left[\frac{\beta^{2}-\gamma^{2}}{\sqrt{1+4 \gamma^{2}}-2 n_{r}-1}+\frac{\sqrt{1+4 \gamma^{2}}-2 n_{r}-1}{4}\right]^{2}
$$

Thereby, if the parameters $\beta, \gamma, \widetilde{\delta}$ are considered into Eq.(4.16), the obtained expression for energy eigenvalue in $l$-state will be same with expression Eq.(3.17) which was obtained by NU method. It should be noted that the same conditions for $n_{r}$ and $V_{0}$ in Eqs.(3.14)-(3.16) obtained by NU method are also determined from the following inequalities $B>0, A<0$. Thus, the determined conditions for $n_{r}, V_{0}$ and $E_{n_{r} l}^{D)}$, i.e., if the conditions the Eqs.(3.14)(3.16) and $V_{e f f, \min }<E_{n_{r} l}^{D)}<V_{l}$ are satisfied respectively, there are the bound states in the system, and the energy spectrum of these states is limited number. According to the SUSY QM metod, knowing the ground state eigenvalues $E_{0}^{(D)}$ and eigenfunctions $u_{0}(r)$, all energy eigenvalues $E_{n_{r} l}^{(D)}$ and eigenfunctions $u_{n_{r} l}(r)$ will be readily obtain. So, the obtained solution of hyper - radial Schrödinger equation by using the eigenfunction of the ground state is exactly equal with the solution obtained by using NU method.

\section{RESULTS AND DISCUSSION}

In this chapter, in order to analyze the present qualitative findings, the single particle energy levels, the effective potentials and normalized hyper - radial wave functions of neutron moving under the average potential field of the ${ }^{56} \mathrm{Fe}$ nucleus in the form the generalized Woods-Saxon potential field, are calculated for various values of $n_{r}$ and $l$ quantum numbers by using the empirical values $r_{0}=1.285 \mathrm{fm}$ and $a=0.65 \mathrm{fm}$ taken from Ref. [62]. Under these certain circumstances, the potential depth of mentioned potential is $V_{0}=(40.5+$ $0.13 \mathrm{~A}) \mathrm{MeV}=47.78 \mathrm{MeV}$, and the radius of the nucleus is $R_{0}=r_{0} A^{\frac{1}{3}}=4.9162 \mathrm{fm}$. Here $A$ is the atomic mass number of ${ }^{56} \mathrm{Fe}$ nucleus. The reduced mass consists of neutron mass $m_{n}=1.00866 u$ and ${ }^{56} \mathrm{Fe}$ core mass with is $m_{A}=56 u$, and its value is $\mu=\frac{m_{A} \cdot m_{n}}{m_{A}+m_{n}}=$ $0.990814 u$.

It would be noted that the dependent term on the orbital quantum number $l$ is as $\tilde{l}(\tilde{l}+1)$ in the energy spectrum expresion $E_{n_{r} l}^{(D)}$ the $\mathrm{Eq}(3.17)$, and $\widetilde{l}(\widetilde{l}+1)$ is invariant for given $N$ positive numbers because of the conversion $l^{\prime}=l \pm N$ and $D^{\prime}=D \mp 2 N$, i.e. $\widetilde{l^{\prime}}\left(\widetilde{l^{\prime}}+1\right)=\widetilde{l}(\widetilde{l}+1)$ based

on the following conversion: $\widetilde{l^{\prime}}=l^{\prime}+\frac{D^{\prime}-3}{2}=l \pm N+\frac{D \mp 2 N-3}{2}=l \pm N+\frac{D-3}{2} \mp N=l+\frac{D-3}{2}=\tilde{l}$. Therefore, when $\left(n_{r}, l, D\right) \longrightarrow\left(n_{r}, l \pm N, D \mp 2 N\right)$, the others energy spectrum in high $D$ 
dimension can be found as $E_{n_{r} l}^{(D)}=E_{n_{r} ; l \pm N}^{(D \mp 2 N)}$ with knowing the energy spectrum in certain dimensions. When $D \geq 2, N$ is equal 1 . Hence, it is sufficient to find the energy spectrum in $D=2$ and $D=3$, because the energy spectrum in high $D$ dimension can be determined by using the following relation: $\quad E_{n_{r} l}^{(D)}=E_{n_{r} ; l \pm 1}^{(D \mp 2)}$, i.e.,

$$
E_{n_{r} ; l}^{(4)}=E_{n_{r} ; l+1}^{(2)}, \quad E_{n_{r} ; l}^{(6)}=E_{n_{r} ; l+2}^{(2)}, \quad E_{n_{r} ; l}^{(8)}=E_{n_{r} ; l+3}^{(2)}, \ldots
$$

and

$$
E_{n_{r} ; l}^{(5)}=E_{n_{r} ; l+1}^{(3)}, \quad E_{n_{r} ; l}^{(7)}=E_{n_{r} ; l+2}^{(3)}, \quad E_{n_{r} ; l}^{(9)}=E_{n_{r} ; l+3}^{(3)}, \ldots
$$

In the case of $D=2$ and $D=3$, the minimum point $r_{\min }$ of effective generalized WoodsSaxon potential and its minimum value $V_{e f f, \text { min }}$, the value $V_{l}=-V_{0}+\frac{\hbar^{2} \tilde{l}(\tilde{l}+1)}{2 \mu R_{0}^{2}}\left(C_{0}+C_{1}+C_{2}\right)$, the bound state energies and the corresponding normalized hyper-radial wave functions are given for certain numbers $l$ and $n_{r}$ in the Table 1 and Table 2, respectively. Additionally, when $D=2$ and $D=3$, the normalized hyper-radial wave functions of the bound state as a function of $z$ are plotted for certain numbers $l$ and $n_{r}$ at $W=100 \mathrm{MeV}$ in Fig. 1 and Fig. 2 , respectively.

It seen from the Table 1 and Table 2 that when $W=100 \mathrm{MeV}$ and $W=200 \mathrm{MeV}$, there are respectively bound states for $n_{r}=0$ in $0 \leq l \leq 5$ and $0 \leq l \leq 6$, but there are not respectively bound states in $l \geq 6$ and $l \geq 7$ as well as when $n_{r} \geq 1$, there are not bound states in any $l$. Furthermore, it seen also from the Table 1 and Table 2 that while the value of $W$ increases, the minimum point of potential $r_{e}$ gets closer to $R_{0}$. Besides, while the orbital quantum number $l$ increases, the energy of bound states $E_{n_{r} l}^{(D)}$ also increase in the setted same value of the radial quantum number $n_{r}$ and $D$ parameter as well as the increasing of $D$ dimension leds to the increasing of bound state energies, i.e., $E_{01}^{(3)}>E_{0 l}^{(2)}, E_{02}^{(3)}>E_{02}^{(2)}, E_{03}^{(3)}>E_{03}^{(2)}$.

It means that, in both cases, there is occurring the pushing force at the expense of the additional centrifugal potential as $V_{l}(r)=\frac{\hbar^{2} \tilde{l}(\tilde{l}+1)}{2 \mu r^{2}}$. Therefore, both the number and value of the energy bound state must increase in order to compensate for this potential [19, 63]. It ought to be noted that the inequality (3.14) and (3.16) are satisfied, but the inequality (3.15) are not satisfied for $n_{r}=1$ and $1 \leq l \leq 5$ case in $W=100 \mathrm{MeV}$ value in Table 1 . It means any of these findings for $n_{r}=1$ and $1 \leq l \leq 5$ cannot be considered as a physical and are only the mathematical results. It has also been determined that when $W=200 \mathrm{MeV}$, there are not bound states for $n_{r}=1$ and $0 \leq l \leq 6$. Hence, in $W=100 \mathrm{MeV}$, and 
$W=200 \mathrm{MeV}$, the non-obtaining bound states of hyper-radial Schrödinger equation for the generalized Woods-Saxon potential in $n_{r}=1$ and $1 \leq l \leq 5$, and $n_{r}=1$ and $0 \leq l \leq 6$, cases can only be eliminated by using the spin and pseudospin symmetry [52 57] in this type potentials.

\section{CONCLUSION}

To conclude, an analytical study of the hyper-radial Schrödinger equation have been performed for the generalized Woods-Saxon potential by using the improved approximation scheme to the centrifugal term for any $l$-states. There the energy eigenvalues of the bound states and corresponding eigenfunctions have been analytically found via both of NU and SUSY QM methods within the Pekeris approximation. The same expressions were obtained for the energy eigenvalues as well as the expression of hyper-radial wave functions transformed each other was shown by using mentioned methods. The energy eigenvalues depending on $V_{0}, W, R_{0}, a$ and $D$ parameters have a finite number energy spectrum for the generalized Wood-Saxon potential, so it puts some limitation on the potential parameters during the solution of the bound states within the framework of quantum mechanics. In this way, if the parameters $V_{0}, n_{r}$ and energy eigenvalues $E_{n_{r} l}^{(D)}$ satisfy the conditions in Eqs.(3.14) - (3.16) and $V_{e f f, \min }<E_{n_{r} l}^{(D)}<V_{l}$ respectively, it means there are the bound states in system. It ought to be noted that the same limiting conditions were obtained for $V_{0}$ and $n_{r}$ thanks to both methods. Since there is the practical interest for the energy spectrum in various potential, investigating the features of eigenvalues is very important and actual with regard to arbitrary parameter of system. For illustration, the bound state energies and corresponding normalized hyper-radial wave functions of neutron moving under the generalized Woods-Saxon potential field, have been calculated and analyzed for some $l$ and $n_{r}$ values. 
[1] R.D. Woods and D.S. Saxon, Phys. Rev. 95 (1954) 577.

[2] A. Bohr and B. Mottelson, Nuclear Structure (World Scientific, Singapore 1998).

[3] J.M.G. Gomez, K. Kar, V.K.B. Kota, R.A. Molina and J. Retamosa, Phys. Lett. B 567 (2003) 251.

[4] S.E. Massen and C.P. Panos, Phys. Lett. A 246 (1998) 530.

[5] H. Koura, M. Yamada, Nucl. Phys. A 671 (2000) 96.

[6] M.E. Brandan and G.R. Satchler, Phys. Rep. 285 (1997) 143.

[7] G.R. Satchler, Phys. Rep. 199 (1991) 147.

[8] D.T. Khoa, G.R. Satchler and W. von Oertzen, Phys. Rev. C 56 (1997) 954.

[9] I. Boztosun, Phys. Rev. C 66 (2002) 024610.

[10] Y. Kucuk, I. Boztosun, T. Topel, Phys. Rev. C 80 (2009) 054602.

[11] A.K. Zaichenko and V.S. Olkhovsky, Theor. and Math. Phys. 27 (1976) 475.

[12] H. Fakhri and J. Sadeghi, Mod. Phys. Lett. A 19 (2004) 615.

[13] C. Berkdemir, A. Berkdemir and R. Sever, Phys. Rev. C 72 (2005) 027001.

[14] B. Gönül and K. Köksal, Phys. Scr. 76 (2007) 565.

[15] O. Bayrak and E. Aciksoz, Phys. Scr. 90 (2015) 015302.

[16] S. Flügge , Practical Quantum Mechanics, Vol. 1 (Springer, Berlin, 1994).

[17] N. Rosen and P.M. Morse, Phys. Rev. 42 (1932) 210.

[18] L.D. Landau and E.M. Lifshitz, Quantum Mechanics (Pergamon Press, London, 1958).

[19] W. Greiner, Quantum Mechanics, 4th. edn. (Springer, Berlin, 2001).

[20] A.F. Nikiforov and V. B. Uvarov, Special Functions of Mathematical Physics (Birkhäuser, Basel, 1988).

[21] F. Cooper, A. Khare and U. Sukhatme, Phys. Rep. 251 (1995) 267.

[22] D.A. Morales, Chem. Phys. Letters, 394 (2004) 68.

[23] H. Ciftci, R.L. Hall and N. Saad, J. of Phys. : Math. Gen. 36 (2003) 11807.

[24] H. Ciftci, R.L. Hall and N. Saad, J. of Phys. : Math. Gen. 38 (2005) 1147.

[25] H. Ciftci, R.L. Hall and N. Saad, Phys. Lett. A: 340 (2005) 388.

[26] O. Bayrak, I. Boztosun and H. Ciftci, Int. J. Quant. Chem. 107 (2007) 540.

[27] O. Bayrak and I. Boztosun, J. Phys. A 39 (2006) 6955. 
[28] O. Bayrak, G. Kocak and I. Boztosun, J. Phys. A: Math. Gen. 39 (2006) 11521.

[29] H. Ciftci, O. Özer and P. Roy, Chin. Phys. B 21 (2012) 010303.

[30] G. Levai and B.W. Williams, J. Phys. A: Math. Gen. 26 (1993) 3301.

[31] P. Amore, A. Aranda and A. De Pace, J. Phys. A: Math. Gen. 37 (2004) 3515.

[32] V.H. Badalov, H.I. Ahmadov and S.V. Badalov, News of Baku University, 2 (2008) 157.

[33] V.H. Badalov, H.I. Ahmadov and A.I. Ahmadov, Int. J. Mod. Phys. E 18 (2009) 631.

[34] V.H. Badalov, H.I. Ahmadov and S.V. Badalov, Int. J. Mod. Phys. E 19 (2010) 1463.

[35] V.H. Badalov, Journal of Qafqaz University, 2 (2014) 178.

[36] H.I. Ahmadov, C. Aydin, N.Sh. Huseynova and O. Uzun, Int. J. Mod. Phys. E 22 (2013) 1350072.

[37] A.I. Ahmadov, C. Aydin and O. Uzun, Int. J. Modern. Phys. A 29 (2014) 1450002.

[38] H.I. Ahmadov, Sh.I. Jafarzade and M.V. Qocayeva, Int. J. Mod. Phys. A 30 (2015) 1550193.

[39] H.I. Ahmadov, M.V. Qocayeva and N.Sh. Huseynova, Int. J. Mod. Phys. E 26 (2017) 1750028.

[40] S.M. Ikhdair, R. Sever, Int. J. Theor. Phys. 46 (2007) 2384.

[41] S.M. Ikhdair, R. Sever, Int. J. Mod. Phys. C 19 (2008) 1425.

[42] S.M. Ikhdair, R. Sever, Int. J. Mod. Phys. C 19 (2008) 221.

[43] S.M. Ikhdair, R. Sever, Int. J. Mod. Phys. E 17 (2008) 1107.

[44] Editorial Note to Phys. Rev. C 72 (2005) 027001; Phys. Rev. C 74 (2006) 039902 (E).

[45] S.M. Ikhdair and R. Sever, Ann. Phys. 16 (2007) 218.

[46] A. Arda and R. Sever, Int. J. Mod. Phys. C 20 (2009) 651.

[47] A. Arda and R. Sever, Int. J. Mod. Phys. A 24 (2009) 3985.

[48] S.M. Ikhdair, B.J. Falaye, M. Hamzavi and , Chin. Phys. Lett. 30 (2013) 020305.

[49] C.L. Pekeris, Phys. Rev. 45 (1934) 98.

[50] R.L. Greene and C. Aldrich, Phys. Rev. A 14 (1976) 2363.

[51] V.H. Badalov, Int. J. Mod. Phys. E 25 (2016) 1650002.

[52] K.T. Hecht and A. Adler, Nucl. Phys. A 137 (1969) 129.

[53] A. Arima, M. Harvey and K. Shimizu, Phys. Lett. B 30 (1969) 517.

[54] J.N. Ginocchio, Phys. Rev. Lett. 78 (1997) 436.

[55] S.-G. Zhou, J. Meng and P. Ring, Phys. Rev. Lett. 91 (2003) 262501.

[56] J.N. Ginocchio, Phys. Rep. 414 (2005) 165.

[57] H. Liang, J. Meng, S.-G. Zhou, Phys. Rep. 570 (2015) 1. 
[58] J. Avery, Hyperspherical Harmonics. Applications in Quantum Theory (Kluwer, Dordrecht, 1989).

[59] H. Bateman and A. Erdelyi, Higher Transcendental functions, Vol. 2. (McGraw-Hill, New York, 1953).

[60] L.E. Gendenshtein, JETP Lett. 38 (1983) 356.

[61] L.E. Gendenshtein, I.V. Krive, Sov. Phys. Usp. 28 (1985) 645.

[62] C.M. Perey, F.G. Perey, J.K. Dickens and R.J. Silva, Phys. Rev. 175 (1968) 1460.

[63] L.E. Ballentine, Quantum Mechanics (World Scientific Publishing, Singapore, 1998). 


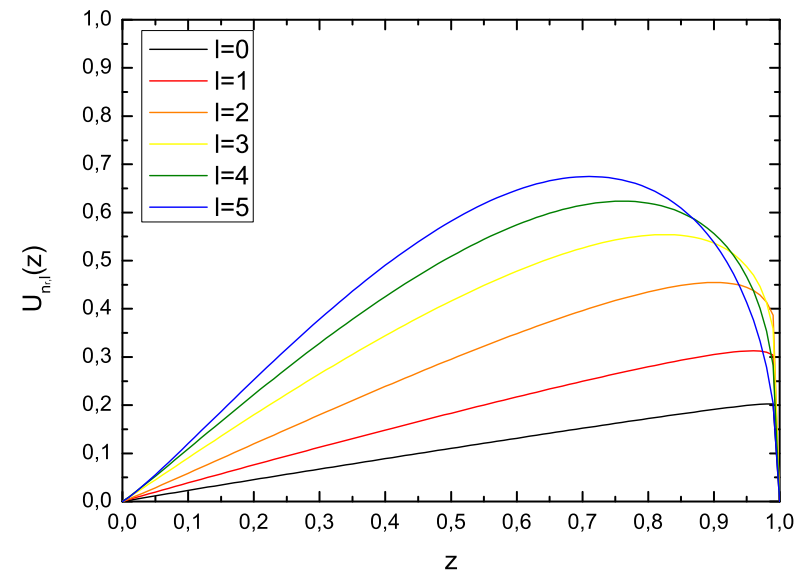

FIG. 1: The normalized hyper-radial wave functions $u_{n_{r} l}(z)$ as a function of $z$ and several quantum numbers $l$ for $V_{0}=47.78 \mathrm{MeV}, R_{0}=4.9162 \mathrm{fm}, a=0.65 \mathrm{fm}, D=2, n_{r}=0$.

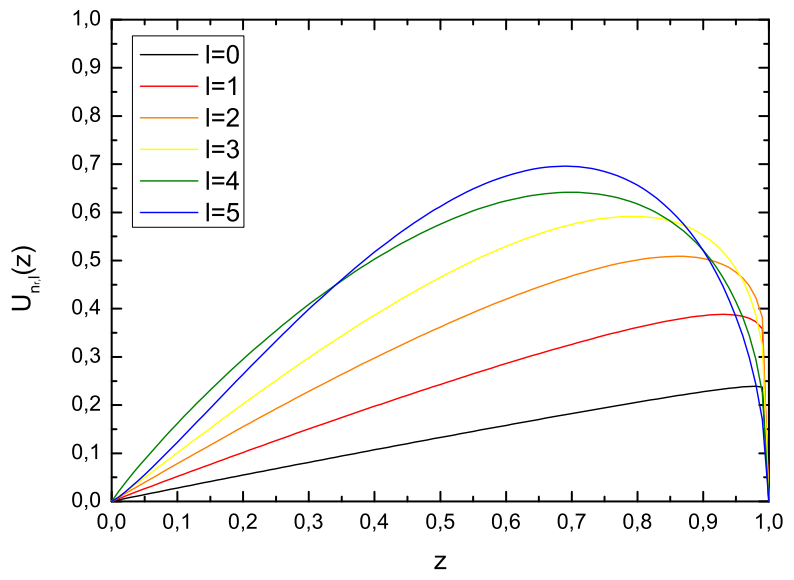

FIG. 2: The normalized hyper-radial wave functions $u_{n_{r} l}(z)$ as a function of $z$ and several quantum numbers $l$ for $V_{0}=47.78 \mathrm{MeV}, R_{0}=4.9162 \mathrm{fm}, a=0.65 \mathrm{fm}, D=3, n_{r}=0$. 


\begin{tabular}{|c|c|c|c|c|c|c|}
\hline$n_{r} \mid l$ & $W, M e V$ & $r_{\min }, f m$ & $V_{\text {effmin }}, M e V$ & $E_{n_{r} l}^{(D)}, M e V$ & $V_{l}, M e V$ & $u_{n_{r} l}(z)$ \\
\hline $0 \mid 0$ & 100 & 4.240031608 & -54.89115601 & -48.28466604 & -48.27312422 & $0.2193602109 z^{0.9767738828}(1-z)^{0.01519140062}$ \\
\hline \begin{tabular}{l|l}
0 & 1
\end{tabular} & 100 & 4.240031608 & -53.71581608 & -46.38731403 & -46.30062733 & $0.3721331375 z^{0.9806163276}(1-z)^{0.04163292552}$ \\
\hline $1 \mid 1$ & 100 & 4.240031608 & -53.71581608 & -23068.47035 & -46.30062733 & Unbound \\
\hline $0 \mid 2$ & 100 & 4.240031608 & -50.18979628 & -41.03113420 & -40.38313667 & $0.6597273740 z^{1.045547623}(1-z)^{0.1138275476}$ \\
\hline $1 \mid 2$ & 100 & 4.240031608 & -50.18979628 & -1016.893447 & -40.38313667 & Unbound \\
\hline $0: 3$ & 100 & 4.240031608 & -44.31309661 & -32.88286462 & -30.52065223 & $0.9860130431 z^{1.027256859}(1-z)^{0.2173302481}$ \\
\hline 13 & 100 & 4.240031608 & -44.31309661 & -218.3838905 & -30.52065223 & Unbound \\
\hline \begin{tabular}{l|l}
0 & 4
\end{tabular} & 100 & 4.240031608 & -36.08571707 & -22.45213564 & -16.71317402 & $1.359442712 z^{1.079307764}(1-z)^{0.3387484872}$ \\
\hline 1 & 100 & 4.240031608 & -36.08571707 & -71.52563286 & -16.71317402 & Unbound \\
\hline \begin{tabular}{l|l}
0 & 5
\end{tabular} & 100 & 4.240031608 & -25.50765766 & -9.98007814 & 1.03929797 & $1.788974091 z^{1.150936539}(1-z)^{0.4693958088}$ \\
\hline 15 & 100 & 4.240031608 & -25.50765766 & -15.77873438 & 1.03929797 & Unbound \\
\hline \begin{tabular}{l|l}
0 & 6
\end{tabular} & 100 & 4.240031608 & -12.57891838 & 4.45561004 & 22.73676374 & Unbound \\
\hline \begin{tabular}{l|l}
0 & 0
\end{tabular} & 200 & 4.599510648 & -76.99336041 & -59.34650380 & -48.20458910 & $1.747348676 z^{1.085457023}(1-z)^{0.4719985004}$ \\
\hline 10 & 200 & 4.599510648 & -76.99336041 & -64.91459392 & -48.20458910 & Unbound \\
\hline \begin{tabular}{l|l}
0 & 1
\end{tabular} & 200 & 4.599510648 & -75.99456066 & -58.16160295 & -46.50623270 & $1.781805687 z^{1.090041485}(1-z)^{0.4827516215}$ \\
\hline \begin{tabular}{l|l}
1 & 1
\end{tabular} & 200 & 4.599510648 & -75.99456066 & -61.50356910 & -46.50623270 & Unbound \\
\hline $0: 2$ & 200 & 4.599510648 & -72.99816142 & -53.74780856 & -41.41116348 & $1.826628107 z^{1.095871056}(1-z)^{0.4966600632}$ \\
\hline $1 \mid 2$ & 200 & 4.599510648 & -72.99816142 & -51.14073127 & -41.41116348 & Unbound \\
\hline 0 & 200 & 4.599510648 & -68.00416269 & -46.81924259 & -32.91938145 & $1.927039632 z^{1.109380285}(1-z)^{0.5271885085}$ \\
\hline $1 \mid 3$ & 200 & 4.599510648 & -68.00416269 & -36.27696168 & -32.91938145 & Unbound \\
\hline 0 & 200 & 4.599510648 & -61.01256446 & -37.35316172 & -21.03088661 & $2.077111816 z^{1.130092659}(1-z)^{0.5712826715}$ \\
\hline $1 \mid 4$ & 200 & 4.599510648 & -61.01256446 & -18.70310516 & -21.03088661 & Unbound \\
\hline $0 \mid 5$ & 200 & 4.599510648 & -52.02336675 & -25.51726932 & -5.74567896 & $2.282942428 z^{1.159069026}(1-z)^{0.6287550789}$ \\
\hline 15 & 200 & 4.599510648 & -52.02336675 & 0.19801712 & -5.74567896 & Unbound \\
\hline \begin{tabular}{l|l}
0 & 6
\end{tabular} & 200 & 4.599510648 & -41.03656954 & -10.78664839 & 12.93624150 & $2.511668721 z^{1.191311742}(1-z)^{0.6887228640}$ \\
\hline $1 \mid 6$ & 200 & 4.599510648 & -41.03656954 & 22.67135873 & 12.93624150 & Unbound \\
\hline \begin{tabular}{l|l}
0 & 7
\end{tabular} & 200 & 4.599510648 & -28.05217283 & 4.69258483 & 35.01487477 & Unbound \\
\hline
\end{tabular}

TABLE I: Calculated the bound state energies and corresponding normalized hyper-radial wave functions for $V_{0}=47.78 \mathrm{MeV}, R_{0}=4.9162 \mathrm{fm}, a=0.65 \mathrm{fm}, \mathrm{D}=2$ in different values of $n_{r}$ and $l$. 


\begin{tabular}{|c|c|c|c|c|c|c|}
\hline$n_{r} \mid l$ & $W, M e V$ & $r_{\min }, f m$ & $V_{e f f m i n}, M e V$ & $E_{n_{r} l}^{(D)}, M e V$ & $V_{l}, \mathrm{MeV}$ & $u_{n_{r} l}(z)$ \\
\hline $0 \mid 0$ & 100 & 4.240031608 & -54.59732103 & -47.80412135 & -47.8 & $0.2654290888 z^{0.9776724433}(1-z)^{0.02196147521}$ \\
\hline \begin{tabular}{l|l}
0 & 1
\end{tabular} & 100 & 4.240031608 & -52.24664116 & -44.10044208 & -43.83500662 & $0.5061434438 z^{0.9862584723}(1-z)^{0.07285183540}$ \\
\hline $1 \mid 1$ & 100 & 4.240031608 & -52.24664116 & -3361.266693 & -43.83500662 & Unbound \\
\hline $0 \mid 2$ & 100 & 4.240031608 & -47.54528142 & -37.26729879 & -35.94501867 & $0.8156347139 z^{1.008916222}(1-z)^{0.1626007103}$ \\
\hline $1 \mid 2$ & 100 & 4.240031608 & -47.54528142 & -429.1546502 & -35.94501867 & Unbound \\
\hline $0: 3$ & 100 & 4.240031608 & -40.49324182 & -27.93239468 & -24.1100373 & $1.166824188 z^{1.050720153}(1-z)^{0.2764562078}$ \\
\hline 13 & 100 & 4.240031608 & -40.49324182 & -123.0701748 & -24.1100373 & Unbound \\
\hline \begin{tabular}{l|l}
0 & 4
\end{tabular} & 100 & 4.240031608 & -31.09052234 & -16.46401658 & -8.33006225 & $1.455628707 z^{0.9352553768}(1-z)^{0.40328453581}$ \\
\hline $1 \mid 4$ & 100 & 4.240031608 & -31.09052234 & -39.07468690 & -8.33006225 & Unbound \\
\hline \begin{tabular}{l|l}
0 & 5
\end{tabular} & 100 & 4.240031608 & -19.33712300 & -3.00613603 & 11.39400663 & $2.031457706 z^{1.193259909}(1-z)^{0.5366086544}$ \\
\hline 15 & 100 & 4.240031608 & -19.33712300 & 2.85638509 & 11.39400663 & Unbound \\
\hline \begin{tabular}{l|l}
0 & 6
\end{tabular} & 100 & 4.240031608 & -5.23304379 & 12.40489010 & 35.06486928 & Unbound \\
\hline \begin{tabular}{l|l}
0 & 0
\end{tabular} & 200 & 4.599510648 & -76.74366047 & -59.04960487 & -47.8 & $1.755963468 z^{1.086598753}(1-z)^{0.4746954280}$ \\
\hline 10 & 200 & 4.599510648 & -76.74366047 & -64.04871484 & -47.8 & Unbound \\
\hline \begin{tabular}{l|l}
0 & 1
\end{tabular} & 200 & 4.599510648 & -74.74606098 & -56.21001797 & -44.38328719 & $1.793100893 z^{1.091444217}(1-z)^{0.4862874478}$ \\
\hline \begin{tabular}{l|l}
1 & 1
\end{tabular} & 200 & 4.599510648 & -74.74606098 & -56.94732674 & -44.38328719 & Unbound \\
\hline $0: 2$ & 200 & 4.599510648 & -70.7508619 & -50.61117817 & -37.58986157 & $1.871017637 z^{1.101800350}(1-z)^{0.5102560391}$ \\
\hline $1 \mid 2$ & 200 & 4.599510648 & -70.7508619 & -44.16267043 & -37.58986157 & Unbound \\
\hline 0 & 200 & 4.599510648 & -64.75806351 & -42.39278162 & -27.39972313 & $1.995480479 z^{1.118763012}(1-z)^{0.5475273550}$ \\
\hline $1 \mid 3$ & 200 & 4.599510648 & -64.75806351 & -27.72415839 & -27.39972313 & Unbound \\
\hline 0 & 200 & 4.599510648 & -56.76766554 & -31.72137742 & -13.81287189 & $2.032995412 z^{1.018948454}(1-z)^{0.5983983356}$ \\
\hline $1 \mid 4$ & 200 & 4.599510648 & -56.76766554 & -9.36267859 & -13.81287189 & Unbound \\
\hline $0 \mid 5$ & 200 & 4.599510648 & -46.77966808 & -18.75894891 & 3.17069217 & $2.408652680 z^{1.176882622}(1-z)^{0.6621806199}$ \\
\hline 15 & 200 & 4.599510648 & -46.77966808 & 9.92379481 & 3.17069217 & Unbound \\
\hline \begin{tabular}{l|l}
0 & 6
\end{tabular} & 200 & 4.599510648 & -34.79407112 & -3.64144188 & 23.55096903 & $2.709803436 z^{1.219329800}(1-z)^{0.7373684670}$ \\
\hline $1 \mid 6$ & 200 & 4.599510648 & -34.79407112 & 29.81656524 & 23.55096903 & Unbound \\
\hline \begin{tabular}{l|l}
0 & 7
\end{tabular} & 200 & 4.599510648 & -20.81087467 & 13.53043692 & 46.32795871 & Unbound \\
\hline
\end{tabular}

TABLE II: Calculated the bound state energies and corresponding normalized hyper-radial wave functions for $V_{0}=47.78 \mathrm{MeV}, R_{0}=4.9162 \mathrm{fm}, a=0.65 \mathrm{fm}, \mathrm{D}=3 \mathrm{in}$ different values of $n_{r}$ and $l$. 